\title{
Numerical Simulation of the Dynamics of a Periodically Forced Spherical Particle in a Quiescent Newtonian Fluid at Low Reynolds Numbers
}

\author{
Tumkur Ramaswamy Ramamohan ${ }^{1}$, Inapura Siddagangaiah Shivakumara ${ }^{2}$, \\ and Krishnamurthy Madhukar ${ }^{1,2}$ \\ ${ }^{1}$ Centre for Mathematical Modelling and Computer Simulation (C-MMACS), Council of \\ Scientific and Industrial Research, Wind Tunnel Road, Bangalore - 560 037, India \\ ${ }^{2}$ UGC-Centre for Advanced Studies in Fluid Mechanics, Department of Mathematics, \\ Bangalore University, Bangalore - 560 001, India \\ trr@cmmacs.ernet.in, isshivakumara@hotmail.com, \\ madhukaracmmacs.ernet.in
}

\begin{abstract}
In this paper we present the results of a numerical simulation of the dynamics of a periodically forced spherical particle in a quiescent Newtonian fluid at low Reynolds number. We describe the simulation and tests performed to validate our simulation. We have obtained results which are physically reasonable and hence we have confidence in our results. We include the effects of both convective and unsteady inertia on the dynamics at low Reynolds numbers. The inclusion of inertia results in additional linear and nonlinear terms in the equations representing a fading memory of the entire history of the motion. The nonlinearity though small in the parametric regime of interest, gives rise to some interesting features in the solution of the problem.
\end{abstract}

Keywords: Low Reynolds numbers, quiescent fluid, spherical particle, periodic force.

\section{Introduction}

The motion of a spherical particle in a fluid at low Reynolds numbers has been of appreciable interest for more than a century, starting with Stokes [1], and his interest on the effects of fluid friction on the motion of pendulums and hence in accurate time keeping. His fundamental expression for the force acting on a spherical particle has motivated many researchers to obtain better approximations for the hydrodynamic force acting on spherical particles. Lovalenti and Brady [2] have summarized work prior to 1993 in their paper and have also derived an expression for the hydrodynamic force undergoing arbitrary time-dependent motion at small Reynolds numbers. In this paper we use the expression derived by Lovalenti and Brady [2] for the hydrodynamic force on a rigid spherical particle undergoing arbitrary time-dependent motion at low Reynolds numbers, to obtain expressions for the particle displacement and particle velocity of a periodically forced spherical particle in a quiescent Newtonian fluid at low Reynolds numbers. 
Our work is motivated by the prior work of Ramamohan and coworkers on the dynamics of periodically forced particles at zero Reynolds numbers i.e., by complete neglect of both unsteady and convective inertia. Kumar and Ramamohan [3] have shown that rheological parameters of suspensions can be controlled using periodic perturbations and that small changes in controllable parameters lead to large changes in rheology. In the present work, our interest is in studying periodically forced suspensions at low Reynolds numbers. The present system is one of the simplest experimentally realizable fluid dynamical systems, at low Reynolds numbers, which can atleast in principle show nonlinear behavior. Further this is an ideal system to investigate fundamental questions about the average behavior of periodically forced systems of nonlinear oscillators and the relationship of the dynamics of a single oscillator to the dynamics of the average behavior of a large number of such oscillators. Here we note that the periodic forcing occurs both at the individual particle level as well as at the level of the averages [4]. This provides a nonlinear coupling between the microlevel of the individual particle and the macrolevel of its averages. In this work we take the first step in the direction of analyzing this problem by determining the effects of a periodic force on the dynamics of a neutrally buoyant spherical particle in a quiescent incompressible Newtonian fluid at low but nonzero Reynolds numbers. This is the simplest possible extension including inertia to the problem studied for over a decade by Ramamohan and coworkers and summarized in Asokan et al. [5]. The inclusion of inertia results in a delay between the variation of the external force and the variation in the response of the particle to the external force. The expression for the hydrodynamic force $F^{H}$ on a spherical particle undergoing an arbitrary motion in an arbitrary time dependent uniform flow field, given by Lovalenti and Brady [2] is used to derive the expression for the particle velocity and displacement using Newton's law and the results are validated by performing several tests on the software. We have generated a number of phase plots (plots between particle displacement and particle velocity) and displacement time series and velocity time series from our simulation. We have analyzed these phase plots and time series using the TISEAN software package, Hegger et al, [6] for nonlinear behavior.

\section{Formulation of the Problem}

The Lovalenti and Brady [2] formalism for the hydrodynamic force on a rigid sphere undergoing arbitrary time - dependent motion in an arbitrary time dependent uniform flow field at small Reynolds numbers is given by the following expression:

$$
\begin{aligned}
& F^{H}(t)=\frac{4 \pi}{3} \operatorname{Re} S l \dot{U}^{\infty}(t)-6 \pi U_{s}(t)-\frac{2 \pi}{3} \operatorname{Re} S l \dot{U}_{s}(t) \\
& +\frac{3}{8}\left(\frac{\operatorname{Re} S l}{\pi}\right)^{1 / 2}\left\{\int _ { - \infty } ^ { t } \left[\frac{2}{3} F_{s}{ }^{H_{11}}(t)-\left\{\frac{1}{|A|^{2}}\left(\frac{\pi^{1 / 2}}{2|A|} \operatorname{erf}(|A|)-\exp \left(-|A|^{2}\right)\right)\right\} F_{s}{ }^{H_{\text {I }}}(s)\right.\right. \\
& \left.+\frac{2}{3} F_{s}^{{ }^{H_{\perp}}}(t)-\left\{\exp \left(-|A|^{2}\right)-\frac{1}{2|A|^{2}}\left(\frac{\pi^{1 / 2}}{2|A|} \operatorname{erf}(|A|)-\exp \left(-|A|^{2}\right)\right)\right\} F_{s}{ }^{H_{\perp}}\right] \\
& \left.\times \frac{2 d s}{(t-s)^{3 / 2}}\right\}+o(\operatorname{Re}) .
\end{aligned}
$$


This expression is obtained by using the reciprocal theorem and the details of the derivation can be found in Lovalenti and Brady [2]. Here, $U_{s}=U_{p}-U^{\infty}$ is the slip velocity of the fluid. $U_{p}$ is the velocity of the particle. $U^{\infty}$ is the velocity of the fluid as $|r| \rightarrow \infty$. Re is the Reynolds number, defined as $R e=a U_{c} / v$ based on a characteristic particle slip velocity, $U_{c}, a$ denotes the characteristic particle dimension and $v$ is the kinematic viscosity of the fluid. $F_{s}{ }^{{ }^{\|}}=-6 \pi U_{s} \cdot \underline{p} \underline{p}$ and $F_{s}{ }^{H_{\perp}}=-6 \pi U_{s} \cdot(\delta-\underline{p} \underline{p})$, where $\delta$ is the idem tensor of order 2 and unit vector $\underline{p}=\frac{Y_{s}(t)-Y_{s}(s)}{\left|Y_{s}(t)-Y_{s}(s)\right|}$, here $Y_{s}(t)-Y_{s}(s)$ is the integrated displacement of the particle relative to the fluid from time $s$ to the current time $t . S l$ is the Strouhal number and $A=\frac{\operatorname{Re}}{2}\left(\frac{t-s}{\operatorname{Re} S l}\right)^{1 / 2}\left(\frac{Y_{s}(t)-Y_{s}(s)}{t-s}\right)$.

For our problem, we consider a neutrally buoyant sphere in an infinite body of a quiescent fluid and consider the effects of an external periodic force acting on the sphere along the $\mathrm{x}-$ axis. We use equation (1) to obtain the equation governing the unidirectional motion of a sphere in a quiescent fluid, starting with zero velocity at time $\mathrm{t}=0$, with $U_{s}=U_{p}$ where $U_{p}$ is the velocity of the particle, scaled with respect to the size of the particle and the frequency of the external periodic force, $\omega$ and $U^{\infty}=0$. Under these conditions, equation (1) reduces to

$$
\begin{aligned}
& F^{H}(t)=-6 \pi U_{p}(t)-\frac{2 \pi}{3} \operatorname{Re} S l \dot{U}_{p}(t) \\
& +\frac{3}{8}\left(\frac{\operatorname{Re} S l}{\pi}\right)^{1 / 2} \int_{0}^{t}\left[\frac{-8 \pi U_{p}(t) d s}{(t-s)^{3 / 2}}-\left\{\frac{1}{|A|^{2}}\left(\frac{\pi^{1 / 2}}{2|A|} \operatorname{erf}(|A|)-\exp \left(-|A|^{2}\right)\right)\right\} \frac{-12 \pi U_{p}(s) d s}{(t-s)^{3 / 2}}\right]
\end{aligned}
$$

We note that the integral in equation (2) contains a singularity at $s=t$. In order to take account of this singularity, the integral was split into the intervals $[0, t-\varepsilon]$ and $[t-\varepsilon, t]$ for a small positive $\mathcal{E}$. We then transform the integral in the interval $[t-\varepsilon, t]$ with respect to $\mathrm{A}$, where

$$
A=\frac{\operatorname{Re}}{2}\left(\frac{t-s}{\operatorname{Re} S l}\right)^{1 / 2}\left(\frac{Y_{s}(t)-Y_{s}(s)}{t-s}\right)
$$

That is, we get,

$$
F^{H}(t)=-6 \pi U_{p}(t)-\frac{2 \pi}{3} \operatorname{Re} S l \dot{U}_{p}(t)+\frac{3}{8}\left(\frac{\operatorname{Re} S l}{\pi}\right)^{1 / 2}(P+Q)
$$

where 


$$
P=\int_{0}^{t-\varepsilon}\left[\frac{-8 \pi U_{p}(t) d s}{(t-s)^{3 / 2}}-\left\{\frac{1}{|A|^{2}}\left(\frac{\pi^{1 / 2}}{2|A|} \operatorname{erf}(|A|)-\exp \left(-|A|^{2}\right)\right)\right\} \frac{-12 \pi U_{p}(s) d s}{(t-s)^{3 / 2}}\right]
$$

and

$$
Q=\int_{t-\varepsilon}^{t}\left[\frac{-8 \pi U_{p}(t) d s}{(t-s)^{3 / 2}}-\left\{\frac{1}{|A|^{2}}\left(\frac{\pi^{1 / 2}}{2|A|} \operatorname{erf}(|A|)-\exp \left(-|A|^{2}\right)\right)\right\} \frac{-12 \pi U_{p}(s) d s}{(t-s)^{3 / 2}}\right]
$$

Transforming the integral with respect to $A$, we get

$$
Q=\int_{0}^{c \sqrt{\varepsilon}} \frac{8 \pi U_{p}^{2}(t) \operatorname{Re} d A}{(\operatorname{Re} S l)^{1 / 2} A^{2}}-\int_{0}^{c \sqrt{\varepsilon}} \frac{1}{\left|A^{2}\right|}\left(\frac{\sqrt{\pi}}{2|A|} \operatorname{erf}(|A|)-\exp \left(-\left|A^{2}\right|\right)\right) \frac{12 \pi U_{p}^{2}(s) \operatorname{Re} d A}{(\operatorname{Re} S l)^{1 / 2} A^{2}}
$$

where ${ }_{c}=\frac{\operatorname{Re} U_{p}(t)}{2 \sqrt{\operatorname{Re} S l}}$. We note that, $Q$ vanishes as $\mathcal{E}$ tends to zero; i.e., as $s \rightarrow t$.

$$
\frac{1}{\left|A^{2}\right|}\left(\frac{\sqrt{\pi}}{2|A|} \operatorname{erf}(|A|)-\exp \left(-\left|A^{2}\right|\right)\right) \rightarrow \frac{2}{3}
$$

Hence the two singular terms cancel each other as $s \rightarrow t$, and thus we obtain an expression for the hydrodynamic force on a sphere in a quiescent fluid as:

$$
\begin{aligned}
& F^{H}(t)=-6 \pi U_{p}(t)-\frac{2 \pi}{3} \operatorname{Re} S l \dot{U}_{p}(t) \\
& +\frac{3}{8}\left(\frac{\operatorname{Re} S l}{\pi}\right)^{1 / 2}\left\{\int_{0}^{t-\varepsilon}\left[\left\{\frac{1}{|A|^{2}}\left(\frac{\pi^{1 / 2}}{2|A|} \operatorname{erf}(|A|)-\exp \left(-|A|^{2}\right)\right)\right\} \frac{12 \pi U_{p}(s) d s}{(t-s)^{3 / 2}}\right]\right. \\
& \left.+16 \pi U_{p}(t)\left[\frac{1}{\sqrt{t}}-\frac{1}{\sqrt{\varepsilon}}\right]\right\}
\end{aligned}
$$

The equation of motion for a neutrally buoyant particle immersed in a fluid is given by

$$
\frac{m_{p} \dot{U}_{p}(t)}{\mu a^{2} \omega}=F^{e x t}(t)+F^{H}(t)
$$

Using equation (4) with the external periodic force $F^{e x t}=F_{0} \sin (t)$, where time has been scaled with respect to the frequency of the external periodic force field, along the $\mathrm{x}$ direction and using Newton's law, we obtain equations for the particle velocity $U_{p}$ and position $Y_{p}$ with velocity and position equal to zero at time equal to zero in the form 


$$
\begin{gathered}
\frac{d Y_{p}}{d t}=U_{p} \\
\frac{d U_{p}}{d t}=\frac{1}{\operatorname{Re}^{*}}\left[\operatorname{Re}_{F} \sin (t)-6 \pi U_{p}+\frac{3}{8}\left(\frac{\operatorname{Re} S l}{\pi}\right)^{1 / 2}\left(J_{1}+I_{1}\right)\right]
\end{gathered}
$$

Here, $\operatorname{Re}^{*}=\frac{4 \pi}{3} \operatorname{Re}+\frac{2 \pi}{3} \operatorname{Re} S l, \operatorname{Re}_{F}=\frac{F_{0}}{\mu a^{2} \omega}, \operatorname{Re}=\frac{\rho a^{2} \omega}{\mu}$, where, $a$ is the particle size, $\omega$ is the frequency of the applied external periodic force, $\mu$ is the viscosity of the fluid and $\rho$ is the density of the particle and the fluid, since the particle is assumed to be neutrally buoyant, we have

$$
J_{1}=16 \pi U_{p}(t)\left[\frac{1}{\sqrt{t}}-\frac{1}{\sqrt{\varepsilon}}\right]
$$

and

$$
I_{1}=\int_{0}^{t-\varepsilon}\left\{\frac{1}{A^{2}}\left(\frac{\sqrt{\pi}}{2|A|} \operatorname{erf}(A)-\exp \left(-A^{2}\right)\right)\right\} \frac{12 \pi U_{p}(s)}{(t-s)^{3 / 2}} d s .
$$

\section{Methodology}

We developed software using Numerical Recipes in FORTRAN [7] to solve equations (5a) and (5b) using an embedded Runge-Kutta method with adaptive step size. The integral in the equation (5b) was evaluated at each time step by Romberg extrapolation. The function with respect to ' $A$ ' was defined by a user supplied function subprogram. We used the ODEINT, RKQS, RKCK subroutines from Numerical Recipes [7] to implement the Runge - Kutta method. The Romberg extrapolation was performed using the QROMB subroutine. The integral was evaluated using TRAPZD and the interpolation during the numerical quadrature was performed by POLINT. The tolerance for both the Romberg extrapolation and the Runge - Kutta solver was taken as $10^{-5}$. Further reduction of the tolerance did not result in any significant change in our results. The entire program was written in double precision. The initial conditions for both the velocity and the position of the particle were taken as zero. $\varepsilon$ was taken as 0.04 ; smaller values of $\varepsilon$ did not significantly change the results. The software was tested for consistency by compiling the program with two compilers namely, Intel Fortran and F90. We generated 5,000 data points taken at an interval of $\pi / 200$ in both the dimensionless velocity and dimensionless position. We note that there are certain novel features of these equations, namely a nonlinear history term that results in certain special features in the solutions.

The results of both the programs matched except for minor differences of the order of $10^{-5}$. In addition to this, we performed the following tests to check the validity of our results. 
TEST1: We performed a perturbation analysis of the problem, using $\operatorname{Re}^{1 / 2}$ as the perturbation parameter. The particle displacement and particle velocity matched with our numerical solutions upto $\mathrm{Re} \approx 0.05$. The deviation from the perturbation solution may be due to the fact that even though Re is small, ' $A$ ' in equation (4), need not be small for all ' $t$ ' and ' $\mathrm{s}$ '. This test is an important test on our software as it showed that the numerical solutions obtained by our software are correct.

TEST2: The solution of the problem of the motion of a spherical particle of greater density than the fluid starting from rest derived using the assumptions of Reynolds number $\mathrm{Re}<<1$ and Strouhal number $S l$ known from the literature ( Fig. 5. of Lovalenti and Brady [2] ) was reproduced.

TEST3: We assumed that the velocity of the fluid at infinity was a constant i.e., $U^{\infty}=U_{0}$ and we set $\operatorname{Re}_{\mathrm{F}}=0$. Under these assumptions, we obtained $U_{p} \rightarrow U_{0}$ as $t \rightarrow \infty$, which is as expected.

TEST4: We generated a number of outputs using $U_{0}=0$ and $U_{0} \neq 0$ and compared the results as $U_{0} \rightarrow 0$. We found that the results matched for $U_{0}=0$ and $U_{0} \rightarrow 0$, up to an order of $10^{-5}$.

TEST5: When we change the initial direction of the motion, namely replacing $\operatorname{Re}_{\mathrm{F}}$ by - $\mathrm{Re}_{\mathrm{F}}$, the phase space plot is reflected about the zero velocity axis. That is, we obtain the reflection of the phase space attractor about the zero velocity axis when the direction of the first motion is reversed. We consider this as an important result which demonstrates the correctness of our results. Our results show a preferred direction in the solution. Since the only physical direction in our problem is the initial direction of the external force, a reversal of that direction should result in a reversal of direction in the solution. We find that this is indeed the case.

TEST6: We observed that there is a shift of position of the attractors when we change the initial condition of $\mathrm{Y}_{\mathrm{p}}$. Changing $\mathrm{Y}_{\mathrm{p}}$ at $\mathrm{t}=0$, results only in a shift of attractors as there is only a change in the frame of reference, which does not affect the physics of the problem. This confirms the fact that a change in the initial position only results in a change in coordinate system and not in any physical parameter. This further increases our confidence in our results.

We note here that, $\mathrm{U}_{\mathrm{p}} \neq 0$ at $\mathrm{t}=0$, does not make any physical sense, since if we consider $\mathrm{U}_{\mathrm{p}} \neq 0$ at $\mathrm{t}=0$, then there must be some particle velocity at negative time too. Moreover, in a quiescent fluid, the particle velocity is due to the application of the external periodic force which is applied only from $t=0$.

These tests give us considerable confidence in our simuation.

\section{Results and Discussions}

Typical phase space plots (plots of particle velocity versus particle position) are plotted in Fig. 1. for different values of $\mathrm{Re}$ and $\mathrm{Re}_{\mathrm{F}}$. We note that these plots represent a bounded region of phase space and hence the plots represent an attractor in phase space. We observe that here, as $\operatorname{Re}_{\mathrm{F}}$ increases the attractor size also increases, 
establishing the obvious relation between the amplitude $\mathrm{Re}_{\mathrm{F}}$ of the forcing term and the size of the attractors. As the amplitude of the periodic force increases, the particle also oscillates with greater amplitude, covering a greater surface area in the attractor plot. One can observe from the figure that as Re increases, the area bounded by attractors decreases, showing the effect of inertia on the motion of the particle. We can observe that for small $\mathrm{Re}_{\mathrm{F}}$, especially for $\mathrm{Re}_{\mathrm{F}}=0.01$ and different $\mathrm{Re}$, the attractors are different from the other attractors shown in Fig. 1. We note here, that near $\operatorname{Re}_{\mathrm{F}}=0.01$, there exists a bifurcation due to the occurrence of a band of higher harmonics or quasi periodicity in the power spectrum and also the presence of kinks at the two extremes of the phase plots as shown in Fig. 1. At low $\mathrm{Re}_{\mathrm{F}}$, the nonlinearity is comparatively large compared to the external periodic force. Re represents the magnitude of the inertial term, namely a resistance to the change in motion. Hence as Re increases, the resistance to the change in motion also increases diminishing the size of the attractors.

The existence of higher harmonics or quasi periodicity is found in these regimes in the power spectrum. We observe that the power spectrum plots are coherent with that of the Stokes' flow case, showing the effect of periodic force on the motion of the particle dominating over the other parameters. However the phase plots in Fig. 1. follow a small spiral, which is due to the presence of damping term in the expression (5b). One can also note that there is a slight drift in the initial motion of the particle as shown in Fig. 1. From this figure it is evident that there are kinks at the two extremes in the phase space plots which occur around zero magnitudes of the velocity of the particle and hence relatively inertial (nonlinear) effects, since near zero velocity, the rate of change of the velocity is highest. On increasing the resolution of our calculations and hence increasing the sampling frequency, these kinks do not change showing the effects of nonlinear term dominating near $\operatorname{Re}_{\mathrm{F}}=0.01$. Typically, these kinks increase in magnitude at large Re.

When we apply a phase shift of $\pi$ to the sinusoidal forcing term the attractors shift their direction. That is, when we apply a force in an initially negative direction (the opposite direction) $Y_{p}$ shows a reflection about the $Y_{p}=0$ axis and thus we obtain a reflection of the attractor. The reflection property of these attractors is evident upon reversing the direction of first motion indicating that these features are not a numerical artefact. Figure 2 show the phase plots when the direction of the amplitude of the force is changed and the attractors form a reflection of each other about the axis $Y_{p}=0$, as expected. Since the direction of the force represents the direction of initial motion and also there is a fading memory, the particle shows an initial displacement and at large times the periodic motion manifests itself, approximately.

We compared the results of the problem with the Stokes' flow results. We have considered the amplitude ratio as the ratio of the amplitude of the motion of the particle in our problem with the Stokes' flow amplitude. We note that except for $\operatorname{Re}_{\mathrm{F}}$ $=0.01$ and higher $\mathrm{Re}$, the amplitude ratio with respect to $\operatorname{Re}$ and $\operatorname{Re}_{\mathrm{F}}$ shows a decreasing trend. This might be due to the effect of inertia of the fluid. In the regime of $\operatorname{Re}_{\mathrm{F}}=0.01$, we observe a greater dependence of the amplitude ratio on $\mathrm{Re}$ and $\mathrm{Re}_{\mathrm{F}}$. This is reasonable since we see a greater effect of nonlinearity in this region. We also observe that as $\operatorname{Re}$ increases the amplitude ratio decreases, showing the effect of inertia is to reduce the amplitude of the motion. 

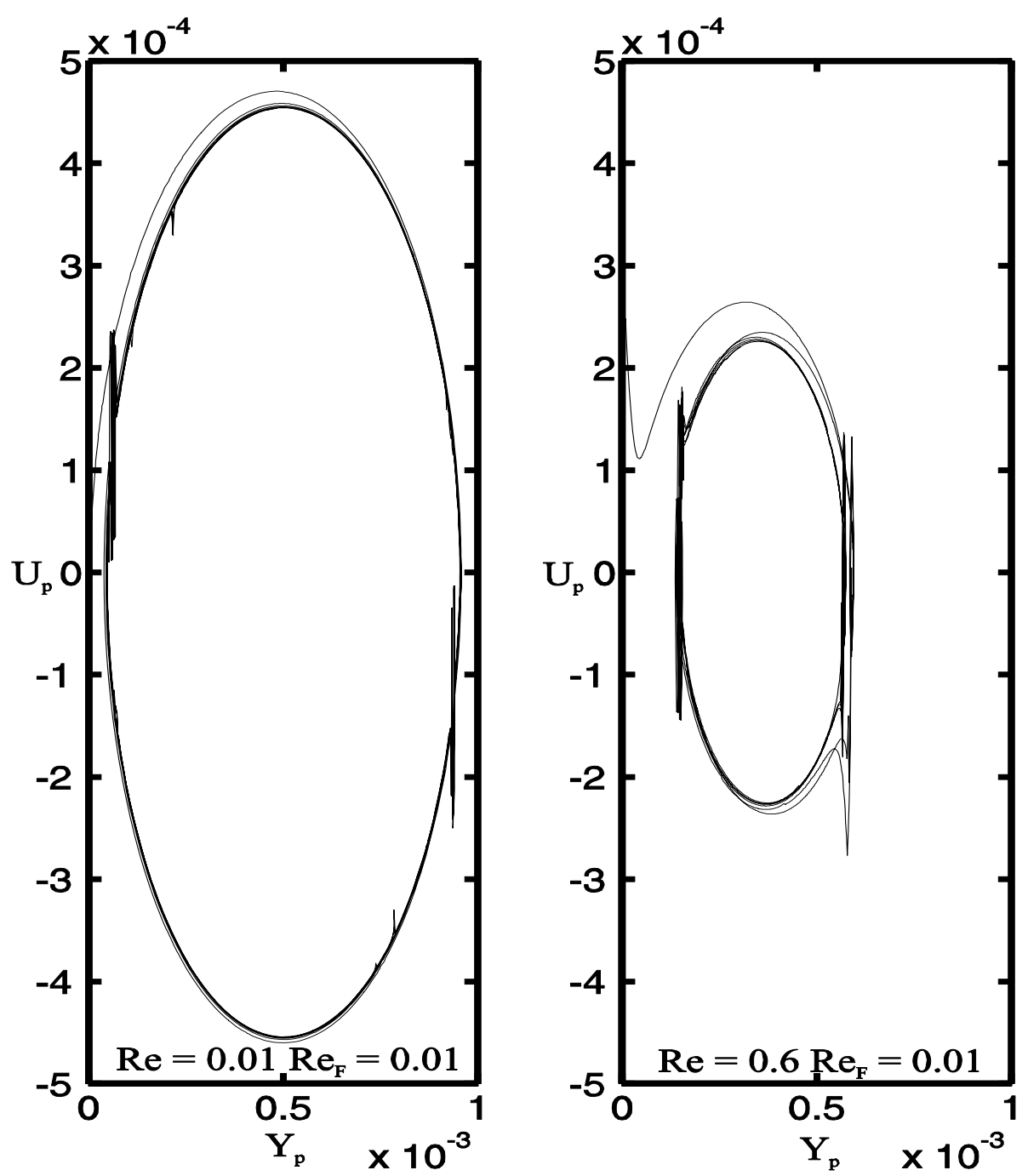

Fig. 1. The phase portrait for various $\mathrm{Re}$ and low $\mathrm{Re}_{\mathrm{F}}$. These attractors show kinks at the two extremes for $\operatorname{Re}_{\mathrm{F}}=0.01$, clearly showing the effect of the nonlinearity, namely a bifurcation near $\operatorname{Re}_{\mathrm{F}}=0.01$.

We observed that there is a definite relationship between the mean particle displacement $Y_{p, \text { mean }}$ and the amplitude of the external periodic force $\operatorname{Re}_{\mathrm{F}}$. This is as expected since the magnitude of the initial motion is determined by the amplitude of the periodic external force and by the value of Re. As Re increases we note that $\mathrm{Y}_{\mathrm{p} \text {,mean }}$ decreases and as $\mathrm{Re}_{\mathrm{F}}$ increases $\mathrm{Y}_{\mathrm{p} \text {,mean }}$ increases.

We obtained the relationship between $\mathrm{Re}, \mathrm{Re}_{\mathrm{F}}$ and amplitude of the velocity of the particle. We observe that the amplitude of the velocity increases with increase in $\operatorname{Re}_{\mathrm{F}}$ and decreases with increase in $\mathrm{Re}$, describing the effect of the periodic force and 
inertia and effect on the amplitude of the velocity of the particle. Using TISEAN [6], we performed tests for the possibility of chaos in the system and found that there occurs a bifurcation at low $\operatorname{Re}_{\mathrm{F}}$ and high $\mathrm{Re}$, there is no chaos in the system.

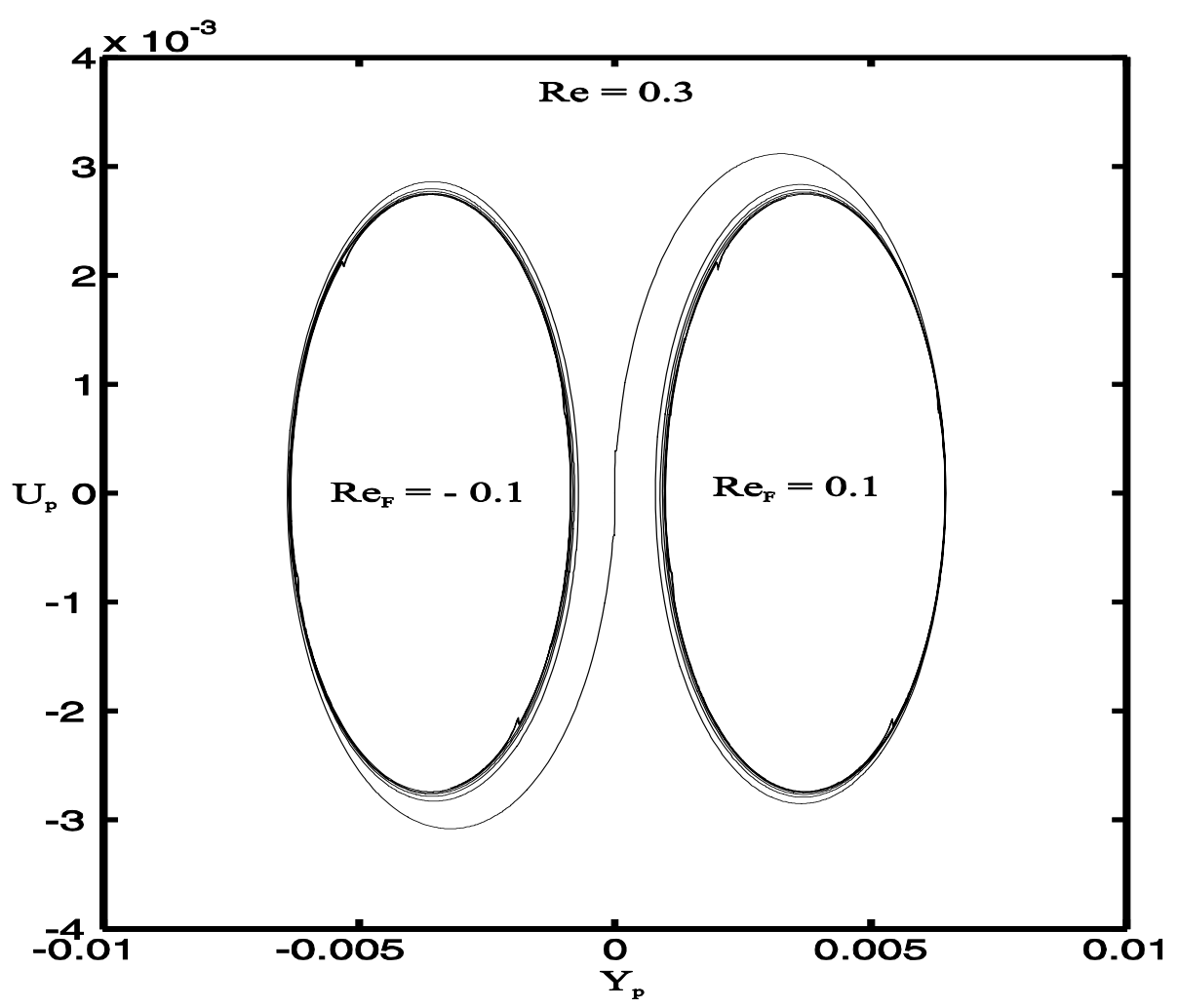

Fig. 2. The phase portrait obtained at $R e=0.3$ and $\operatorname{Re}_{F}= \pm 0.1$. The phase portrait shows the reflection property of the solutions of our problem, indicating that there is a physical basis to our results.

\section{Conclusion}

In this paper, we have attempted to determine the effects of a periodic force on a sphere in a quiescent fluid at low Reynolds numbers. We observe that the particle oscillates around a mean position, due to the periodic force on the particle. There is a net displacement of the mean position of the particle in the direction of first motion. The presence of higher harmonics in the full problem for small $\mathrm{Re}_{\mathrm{F}}$ and high $\mathrm{Re}$ are noticed and this shows that though there is a nonlinear term in the equation, its effect is small in the parametric regime which we have considered except at low values of $\mathrm{Re}_{\mathrm{F}}$ and large values of Re. It is also observed that increasing Re was responsible for an increase in the resistance to the change in particle motion and hence a decrease in attractor area and increasing $\operatorname{Re}_{\mathrm{F}}$ leads to an increase in the amplitude of the motion of 
the particle. Besides, a reflection of the attractor on changing the initial direction of motion is obtained. Our results of the dependence of the mean position of the particle and the amplitude of the velocity of a particle on the problem parameters, such as $\mathrm{Re}$ and $\mathrm{Re}_{\mathrm{F}}$ may be used to estimate appropriate physical parameters of the system by suitable experiments. We hope that this work will excite further interest in this area.

\section{Acknowledgements}

The authors wish to acknowledge Dr. A R Upadhya, Scientist - in - Charge, CSIRCMMACS, Bangalore-560037, India and Prof. N. Rudraiah, Honorary Professor, UGC - CAS, Department of Mathematics, Bangalore University, Bangalore 560001, India, for their kind encouragement. The authors also wish to acknowledge the Department of Science and Technology, Govt. of India, New Delhi- 110016, India for financial assistance vide Sanction Letter No. SR/S3/CE/33/2004-SERC Engg. One of the authors, K. Madhukar wishes to thank NAL/C-MMACS for providing the fellowship to do this research work.

\section{References}

1. Stokes, G.G.: On the effect of internal friction of fluid on the motion of pendulums. Trans. Camb. Phil. Soc. 9, 8-106 (1851)

2. Lovalenti, P.M., Brady, J.F.: The hydrodynamic force on a rigid particle undergoing arbitrary time - dependent motion at small Reynolds numbers. J. Fluid Mech. 256, 561-605 (1993)

3. Kumar, C.V.A., Ramamohan, T.R.: Controlling chaotic dynamics of periodically forced spheroids in simple shear flow: Results for an example of a potential application. Sadhana 23, 131-149 (1998)

4. Radhakrishnan, K., Asokan, K., Dasan, J., Bhat, C.C., Ramamohan, T.R.: Numerical evidence for the existence of a low dimensional attractor and its implications in the rheology of dilute suspensions of periodically forced slender bodies. Phys. Rev. E. 60, 6602-6609 (1999)

5. Asokan, K., Kumar, C.V.A., Dasan, J., Radhakrishnan, K., Kumar, K.S., Ramamohan, T.R.: Review of chaos in the dynamics and rheology of suspensions of orientable particles in simple shear flow subject to an external periodic force. J. Non-Newtonian Fluid Mech. 129, 128-142 (2005)

6. Hegger, R., Kantz, H., Schreiber, T.: Practical implementation of nonlinear time series methods. CHAOS 9, 413-435 (1999)

7. Press, W.H., Teukolsky, S.A., Vetterling, W.T., Flannery, B.P.: Numerical recipes in Fortran. In: The art of scientific computing, 2nd edn. Cambridge University Press, Cambridge (1992) 